\title{
Influence of peripheral corneal relaxing incisions during cataract surgery for corneal astigmatism up to 2.5 dioptres on corneal densitometry
}

\author{
leva Zukaite ${ }^{1,2} \cdot$ Kaveeta K. Bedi $^{1} \cdot$ Shahnaz Ali ${ }^{3}$ Mayank A. Nanavaty ${ }^{1,3}$
}

Received: 19 May 2018 / Revised: 14 October 2018 / Accepted: 11 December 2018 / Published online: 2 January 2019

(c) The Royal College of Ophthalmologists 2019

\begin{abstract}
Purpose: To assess the effect of peripheral corneal relaxing incisions (PCRI) for astigmatism between 0.75 and 2.5 dioptres during cataract surgery on corneal densitometry (CD).

Methods: In this prospective, randomised study, 80 eyes (80 patients), received either tIOL or PCRI. Assessment at preoperative and 1, 3, 6, 12 months post-operative visit included uncorrected (UCDVA) and best-corrected distance visual acuity (BCDVA), spherical equivalent (SEQ) (only post-operatively), mean anterior and posterior keratometric astigmatism $\left(K_{\mathrm{A}}\right)$ and $\mathrm{CD}$ with Schiempflug system. $\mathrm{CD}$ was analysed in four concentric radial zones from centre to periphery (Zone 1 to 4) and in 3 layers (anterior, mid-stromal and posterior).

Results: Comparing tIOLs vs. PCRIs, there was no significant difference in the UCDVA, BCDVA and SEQ. In PCRI group, anterior $K_{\mathrm{A}}$ decreased at 1 month and remained stable thereafter. For zones 1,2 and 3, CD significantly reduced after 3 months with tIOLs whereas with PCRIs, it reduced 1 month onwards. For zone 4, CD reduced only at 12 months with tIOLs compared to 3 months onwards with PCRIs. In both groups CD was higher in the zone 4 and anterior layer. Significant reduction in CD was found in all three layers of cornea after 3 months in tIOL and after first month in PCRI groups, respectively.

Conclusions: Cataract surgery alone reduces the CD. Reducing keratometric astigmatism with PCRIs shows significant differences in CD from early post-operative period for central and anterior corneal layer.
\end{abstract}

\section{Introduction}

The transparency of cornea is a result of precise organisation of stromal collagen fibres and extracellular matrix [1, 2]. Some studies describe corneal densitometry (CD) as an objective indicator of corneal health [3,4], which is affected

Presentation: Submitted for presentation at the European Society of Cataract \& Refractive Surgery meeting, Vienna, September 2018.

Mayank A. Nanavaty

mayank.nanavaty@bsuh.nhs.uk

$1 \quad$ Sussex Eye Hospital, Brighton \& Sussex University Hospitals NHS Trust, Eastern Road, Brighton BN2 5BF, UK

2 Medical Faculty, Otto-von-Guericke-University Magdeburg, 39120 Magdeburg, Germany

3 Brighton \& Sussex Medical School, University of Sussex, Falmer, Brighton BN1 9PX, UK by a natural aging process [5-7]. The organised network together with other factors, such as intraocular pressure or the action of extraocular muscles [8], affect the mechanical strength and stability of the cornea. Nevertheless, the biomechanics of the tissue can be modified by various medical procedures, such as corneal refractive surgery [9].

Many methods have been used to evaluate the changes in corneal structure. The slit lamp examination of anatomical eye structures allows detection of gross changes such as corneal haze. However, this is a subjective examination method. Freund proposed a method of direct summation of field for light scattering [10] but, due to its complexity, the measuring principle was not established. Smith first stated the method of Scheimpflug imaging technique in an article in 1990 [11]. Currently this same imaging technique is used in Pentacam $\mathrm{HR}^{\circ}$ (Oculus Optikgeräte $\mathrm{GmbH}$, Germany) to provide a quantitative information of corneal tissue and the objective and non-invasive evaluation of corneal transparency and CD. To the best of our knowledge, there are no studies on effect of cataract surgery on $\mathrm{CD}$ and how does this $\mathrm{CD}$ change if 
cataract surgery is combined with a refractive procedure like a peripheral corneal relaxing incision (PCRIs).

The purpose of this study was to observe whether cataract surgery with peripheral corneal relaxing incisions (PCRI) for correction of keratometric astigmatism between 0.75 and 2.5 dioptres has an additional impact on changes in comparison to cataract surgery with toric intraocular lens (tIOL) without any PCRIs.

\section{Methods}

This prospective randomised clinical trial was conducted in adherence to the tenets of Declaration of Helsinki and was approved by the National Ethics Committee. The study was carried out at the Brighton and Sussex University Hospitals NHS Trust, Brighton, England, between March 2014 and May 2015. Registration of this study was accomplished with UKCRN (ID: 16848) and www.Clinicaltrials.gov (ID: NCT02067429). All participants were enroled from the cataract pre-assessment clinics. Written informed consent was obtained from all patients before the surgical procedures at the beginning of the study. Participants were free to abandon the study at any point, without having to give any reason. The first eye of those with cataract, corneal astigmatism between $\geq 0.75 \mathrm{D}$ and $\leq 2.5 \mathrm{D}$ on topography (Pentacam $\mathrm{HR}^{\circ}$, Oculus, Germany) and post-operative visual potential of $0.2 \operatorname{LogMAR}$ or better, were included in the study. The exclusion criteria were as follows: diabetes, glaucoma, astigmatism outside the study range, abnormal corneal topography or any other co-existing retinal or cornea conditions, concurrent use of ocular medications including lubricants. Patients that were unable to consent or attend follow-up appointments were also excluded.

\section{Randomisation}

Eighty eyes of 80 patients were recruited for assessment: 40 in each group (tIOL and PCRI). The eyes were randomly sorted to either intervention group (tIOL or PCRI) by using a random number generator created by a member of the Research and Development team who did not participate in assessment or surgery. Information about the required procedure (tIOL or PCRI) was given to the research team just before the patient attended a surgery in the operating room. The team provided a blocked randomisation sequence (blocks of random size 2, 4 and 6). Two different envelopes were generated to guarantee a distribution of patients over total corneal astigmatism range of $0.75-2.5 \mathrm{D}$. One consisted of 80 randomisation allocation codes for participants with $0.75-1.5$ dioptres and the other contained 80 randomisation allocation codes for patients with 1.5-2.5 dioptres. Despite including just the first eye of the patient in the study, the second eye underwent the same intervention (tIOL or PCRI) as the first, but using a normal NHS protocol. Patients requiring toric IOLs had T-flex Rayner and PCRI group received Cflex Rayner IOLs (Rayner Intraocular Lenses, Worthing).

\section{Surgical procedures}

A single experienced right-handed surgeon (MN) performed all surgeries under topical anaesthesia as a routine procedure. Prior to surgery, a drop of G. Proxymethacaine (Minims, Bausch \& Lomb, Rochester, USA) was instilled and the eye was marked during slit lamp examination. Beneficial to mark the eye, a Tomark TIOL marker (Geuder, Germany) was fixed to the applanation tonometer and 0 and 180 degrees on the toric marker were aligned to the corresponding degrees on the applanation tonometer. The participant was asked not to blink and requested to fixate on a distant point during the marking procedure. All patients (regardless of tIOL or PCRI) received the marks at 0 and 180 degrees on the slit lamp before the surgery.

The surgical technique and calculation for PCRIs and toric IOLs used in this study are already described elsewhere [12].

\section{Pre-operative and post-operative measurements}

Pre-operative measurements included optical biometry on IOLMaster 500 (Carl Zeiss Meditec, Germany), an ultrasound biometry (Zeiss Meditec, Germany) in case biometry could not be proceeded on IOLMaster 500 because of density of cataract, uncorrected (UCDVA) and bestcorrected distance visual acuity (BCDVA), anterior and posterior keratometric astigmatism $\left(K_{\mathrm{A}}\right)$, mean keratometry $\left(K_{\mathrm{M}}\right)$ and $\mathrm{CD}$ on Pentacam HR ${ }^{\circ}$. CD measurements were taken at pre-operative and 1, 3, 6 and 12 months postoperative appointments. An experienced research technician and the principle investigator (MAN) carried out all the assessments.

\section{Corneal densitometry measurements}

In addition to the corneal topographic assessments, Scheimpflug images on the Pentacam HR ${ }^{\circ}$ provides information on $\mathrm{CD}$. The measurement protocol includes a series of 25 images $(1003 \times 520$ pixels) taken over different meridians with a uniform blue light source. The acquisition protocol takes circa $2 \mathrm{~s}$ to complete. All the scans were taken in the same room with standardised mesopic light for all patients at all visits. During the analysis phase, the programme automatically detects the corneal apex and analyses the $12 \mathrm{~mm}$ diameter area around it. Densitometry values obtained and expressed in standardised greyscale units (GSUs). The GSU scale is calibrated by averages of 


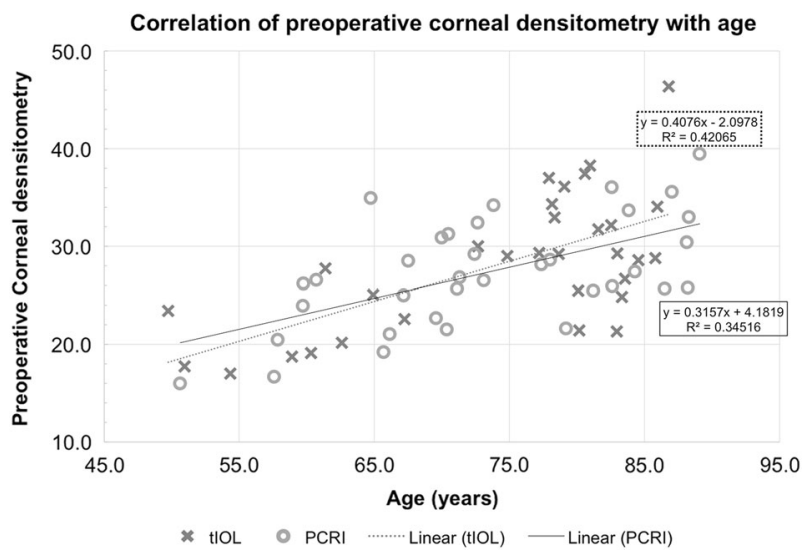

Fig. 1 Correlation of corneal densitometry with age in toric IOL and PCRI groups

proprietary software, which defines a minimum light scatter of 0 (maximum transparency) and a maximum light scatter of 100 (minimum transparency).

In order to proceed to a local densitometry analysis, the measurements were taken in reach of the default $12 \mathrm{~mm}$ diameter area which is subdivided into four concentric radial zones. The corneal apex was indicated as a central point of all zones:

Zone 1: The first central zone is $2 \mathrm{~mm}$ in width.

Zone 2: The second annulus extending from 2 to $6 \mathrm{~mm}$ annulus.

Zone 3: The third zone annulus goes from 6 to $10 \mathrm{~mm}$ in diameter.

Zone 4: The final external annulus from 10 to $12 \mathrm{~mm}$ in diameter.

These topographical zones are established in the software. Furthermore, in the software the CD was analysed in different depths:

a. Anterior layer with $120 \mu \mathrm{m}$ of outermost cornea

b. Mid-stromal layer in between the anterior and posterior layers

c. Posterior layer with thickness of $60 \mu \mathrm{m}$

\section{Statistical analysis}

Statistical analysis was performed with Microsoft Office Excel $^{\bullet} 2016$ (Microsoft $^{\bullet}$ Corporation, USA). The obtained values were statistically analysed and are expressed as mean \pm SD values. Parametric statistical tests were applied to compare variables. The t-test for paired samples was used to compare the pre- and post-operative densitometry values. The ANOVA test was used for evaluation of 1, 3, 6 and 12 months densitometry values. All parameters between the tIOL and PCRI groups were compared at each visit using t-test for independent samples. The level of significance was defined as $P<0.05$.

\section{Results}

Eighty patients were recruited for this study; however, the full follow-up data was available for 70 patients, which were included for further analysis. The study included patients with the age from 50 to 89 years. The mean age at the time of operation was $73.90 \pm 10.60$ years: 31 participants in the tIOL intervention group (13 female and 18 male) and 35 participants in PCRI intervention group (19 female and 16 male). As seen in the graphs (Fig. 1), the $\mathrm{CD}$ values correlated with the age in both tIOL $\left(R^{2}=0.4207\right)$ and PCRI $\left(R^{2}=0.3452\right)$ groups.

\section{(a) Visual acuity, spherical equivalent and keratometry}

There was no significant difference in the UCDVA and BCDVA at 12 months between the groups (Table 1). There was a significant difference only at 1 month, with the PCRI group showing better BCDVA compared to the tIOL group (Table 1). However, there was no difference in UCDVA and BCDVA at all other post-operative visits (Table 1). There was no significant difference in the spherical equivalent within the groups comparing all follow-up visits and between groups at all follow-ups. The post-operative total $\mathrm{CD}$ at 12-months follow-up and 12-month post-operative spherical equivalent showed no correlation in both tIOL and PCRI groups. Preoperatively, comparing the tIOL and PCRI groups, there were no significant differences in mean anterior and posterior $K_{\mathrm{A}}$ and $K_{\mathrm{M}}$. Post-operatively, anterior $K_{\mathrm{A}}$ decreased significantly after surgery until 12 months in participants with PCRIs (Table 1). Further analysis in the PCRI group revealed that the decrease in anterior $K_{\mathrm{A}}$ occurred 1 month post-operatively and remained significantly low. Thereafter, no significant differences were found from months 1-12. No significant differences were found in posterior $K_{\mathrm{A}}$ over the 12 months. Although the posterior $K_{\mathrm{A}}$ and $K_{\mathrm{M}}$ were more negative in the PCRI group, there were no significant differences postoperatively in posterior $K_{\mathrm{A}}$ and $K_{\mathrm{M}}$ between the groups at all follow-up visits.

\section{(b) Corneal densitometry in each corneal ring zone}

There were significant changes in CD after cataract surgery in each tIOL and PCRI groups.

i. For tIOLs: The pre-operative CD (Table 2a) significantly reduced in the corneal zones 1,2 and 3 at 3 , 6 and 12 months and in the zone 4 at 12 months 
follow-up only (Table 2b). Moreover, the values were observed increasingly higher in the zone 4 than in the central zones.

ii. For PCRIs: The CD reduced significantly at all follow-ups in zones 1 and 2; whereas in zone 3 at 1, 3 and 12 months post-operatively and in zone 4 at 3,6 and 12 months (Table 2b). Moreover, the values were observed increasingly higher in the zone 4 than in the central zones.

iii. Comparing tIOLs vs. PCRIs: there was no statistically significant difference $(P>0.05)$ between the interventions in any corneal zones (Table $2 \mathrm{a}$ ).

\section{(c) Corneal densitometry values according to corneal layers}

i. Total corneal thickness: As for total pre- vs. postoperative comparison over the entire corneal thickness, the values significantly reduced at 3, 6 and 12 months for tIOLs and at all four post-operative appointments for PCRIs (Table 2c and Fig. 2).

ii. Anterior corneal layer (anterior $120 \mu \mathrm{m}$ ): In tIOL group, CD significantly reduced at 3, 6 and 12 months post-operative. In PCRI group, it was significantly different at all follow-up visits (Table 2c).

iii. Mid-stromal corneal layer: In tIOL group, CD significantly reduced at 3, 6 and 12 months. In PCRI group, it was significantly different at all follow-up visits (Table 2c).

iv. Posterior corneal layer: In tIOLs, the change was significant at 3 and 12 months only, whereas in the PCRI group, it was significantly different at all follow-up visits (Table 2c).

\section{(d) Overall findings}

In both of the groups, the significant change in $\mathrm{CD}$ was more in anterior and stromal layers compared to posterior layer. The values were found higher in anterior $120 \mu \mathrm{m}$ layer compared to the mid-stromal or posterior $60 \mu \mathrm{m}$ layers. No significant difference in densitometry values was found between both intervention groups (tIOL vs. PCRI) in any corneal layer (Table 2d).

\section{Discussion}

This study found that the cataract surgery with tIOL or PCRI to correct astigmatism up to $2.5 \mathrm{D}$ does reduce CD over 1 year in both the groups. There was no significant

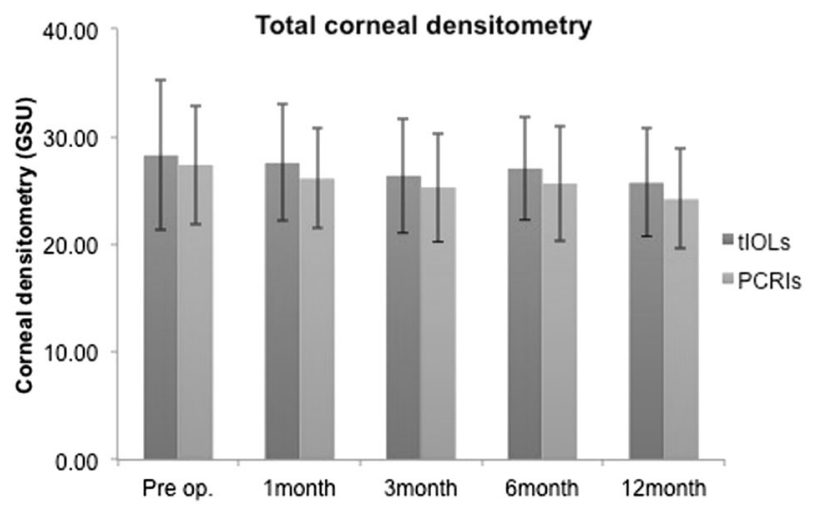

Fig. 2 Total corneal densitometry in both groups over 12 months follow-up

difference between the groups. In both of the groups, CD values change significantly in central corneal zones compared to peripheral and they change more in anterior and mid-stromal layers compared to posterior corneal layer. Significant CD changes were seen 1 month onwards after PCRIs compared to 3 month onwards in tIOL group.

CD has been studied on patients with general systemic diseases such as type 1 diabetes mellitus [13] and rheumatoid arthritis [14] as well as in some ocular conditions like bacterial keratitis [4], Fuchs' endothelial dystrophy [15] and keratoconus [16]. The changes in CD can occur due to various corneal refractive surgeries. Our aim of this study was to assess the $C D$ values in patients undergoing cataract surgery with tIOL or PCRI to correct astigmatism $\leq 2.5 \mathrm{D}$ at 1-year follow-up. Moreover, we were more interested to see if the peripheral relaxing incisions during cataract surgery have an additional impact on CD.

The pre-operative $\mathrm{CD}$ values in tIOL and PCRI groups were $28.26 \pm 6.92$ and $27.35 \pm 5.57$, and at 12 months followup $25.72 \pm 4.99$ and $24.18 \pm 4.63$ in our study. Those CD values were slightly higher than the average of $23.6 \pm 4.47$ found in 70-80 years Caucasian study participants [3], but still are in the normal range, as any value under $\sim 30 \mathrm{GSU}$ is considered as normal CD. Nevertheless, it has to be noted that the mean age of participants in our study was $73.90 \pm 10.60$ years and the corneal morphological structure can be affected by aging, causing increased $\mathrm{CD}$ values in comparison to young healthy people. Like Dhubhgahaill et al. [3] and Alzahrani et al. [17]. we observed a positive correlation in CD with age in our study. One important reason causing less transparent cornea during aging is the loss of endothelial cells with the annual decrease of $0.6 \%$, increase in pleomorphism and polymegathism in people from 20 to 80 years $[18,19]$. Those changes affect the hydration of cornea and the transparency of cornea is the best in its deturgesced state.

In our study, no significant difference in $\mathrm{CD}$ was found between PCRI and tIOL intervention groups (Table 2a). When densitometry was analysed within the groups, the 


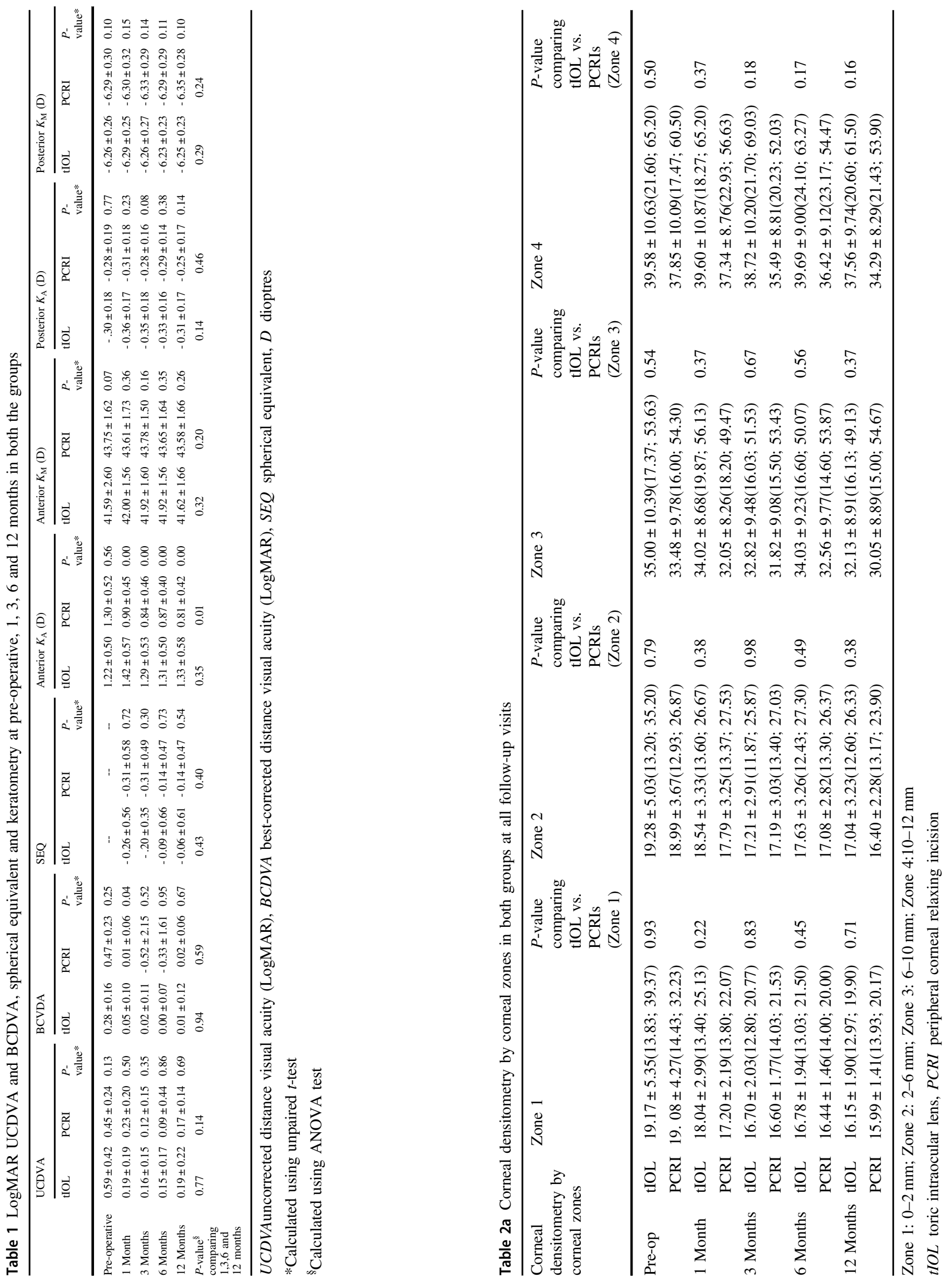


Table 2b $P$-values of comparisons of pre-operative vs. various followup visits in each zones for tIOLs and PCRIs groups

\begin{tabular}{llllll}
\hline Pre-operative vs. & Zone 1 & Zone 2 & Zone 3 & Zone 4 \\
\hline tIOL & 1 Month & 0.3 & 0.49 & 0.4 & 0.92 \\
& 3 Months & $0.01^{\mathrm{a}}$ & $0.01^{\mathrm{a}}$ & $0.02^{\mathrm{a}}$ & 0.3 \\
& 6 Months & $0.01^{\mathrm{a}}$ & $0.01^{\mathrm{a}}$ & $0.007^{\mathrm{a}}$ & 0.1 \\
& 12 Months & $0.001^{\mathrm{a}}$ & $0.01^{\mathrm{a}}$ & $0.001^{\mathrm{a}}$ & $0.02^{\mathrm{a}}$ \\
PCRI & 1 Month & $0.04^{\mathrm{a}}$ & $0.048^{\mathrm{a}}$ & $0.005^{\mathrm{a}}$ & 0.17 \\
& 3 Months & $0.002^{\mathrm{a}}$ & $0.002^{\mathrm{a}}$ & $0.004^{\mathrm{a}}$ & $0.01^{\mathrm{a}}$ \\
& 6 Months & $0.003^{\mathrm{a}}$ & $0.004^{\mathrm{a}}$ & 0.068 & 0.049 \\
& 12 Months & $0.0003^{\mathrm{a}}$ & $0.0003^{\mathrm{a}}$ & $0.0003^{\mathrm{a}}$ & $0.04^{\mathrm{a}}$ \\
\hline
\end{tabular}

Zone 1: 0-2 mm; Zone 2: 2-6 mm; Zone 3: 6-10 mm; Zone 4:10-12 m tIOL toric intraocular lens, PCRI peripheral corneal relaxing incision ${ }^{a}$ Statistically significant

Table 2c $P$-values of comparisons of pre-operative vs. various followup visits in each corneal layers for tIOLs and PCRIs groups

\begin{tabular}{llllll}
\hline Pre-operative vs. & $\begin{array}{l}\text { Anterior } \\
120 \mu \mathrm{m}\end{array}$ & $\begin{array}{l}\text { Mid- } \\
\text { stromal }\end{array}$ & $\begin{array}{l}\text { Posterior } \\
60 \mu \mathrm{m}\end{array}$ & $\begin{array}{l}\text { Total } \\
\text { corneal }\end{array}$ \\
\hline \multirow{4}{*}{ tIOL $\quad$ 3 Month } & 0.23 & 0.94 & 0.7 & 0.49 \\
& 6 Months & $0.02^{\mathrm{a}}$ & $0.04^{\mathrm{a}}$ & $0.01^{\mathrm{a}}$ & $0.01^{\mathrm{a}}$ \\
& $0.01^{\mathrm{a}}$ & $0.01^{\mathrm{a}}$ & $0.01^{\mathrm{a}}$ & $0.00^{\mathrm{a}}$ \\
12 Months & $0.00^{\mathrm{a}}$ & $0.00^{\mathrm{a}}$ & $0.00^{\mathrm{a}}$ & $0.00^{\mathrm{a}}$ \\
PCRI & 3 Month & $0.02^{\mathrm{a}}$ & $0.02^{\mathrm{a}}$ & $0.02^{\mathrm{a}}$ & $0.01^{\mathrm{a}}$ \\
& 6 Months & $0.00^{\mathrm{a}}$ & $0.00^{\mathrm{a}}$ & $0.00^{\mathrm{a}}$ & $0.00^{\mathrm{a}}$ \\
& $0.00^{\mathrm{a}}$ & $0.00^{\mathrm{a}}$ & $0.02^{\mathrm{a}}$ & $0.00^{\mathrm{a}}$ \\
\hline
\end{tabular}

$t I O L$ toric intraocular lens, PCRI peripheral corneal relaxing incision

${ }^{a}$ Statistically significant

values were higher in the anterior corneal layer and topographically in the peripheral 10-12 $\mathrm{mm}$ annulus (Table 2a, d). Same variation in densitometry values was observed in few other studies $[3,20,21]$. When the total $C D$ values were compared topographically, we found that PCRI does reduce the values in peripheral $10-12 \mathrm{~mm}$ diameter annulus more than just tIOL intervention (Table 2a). However, these results were not significant. The Scheimpflug camera scans the entire anterior segment of the eye, from the anterior surface of the cornea to the posterior surface of the lens [22]. We hypothesise, that the significant reduction of $\mathrm{CD}$ in the central corneal zones $(0-6-\mathrm{mm})$ in both the groups following cataract surgery could be due to reduced backscatter of light.

Changes in CD have been studied also in other corneal refractive procedures. Current research has shown corneal transparency changes after corneal collagen cross-linking (CXL) [23, 24] and various refractive surgeries: photorefractive keratectomy (PRK), laser-assisted in situ

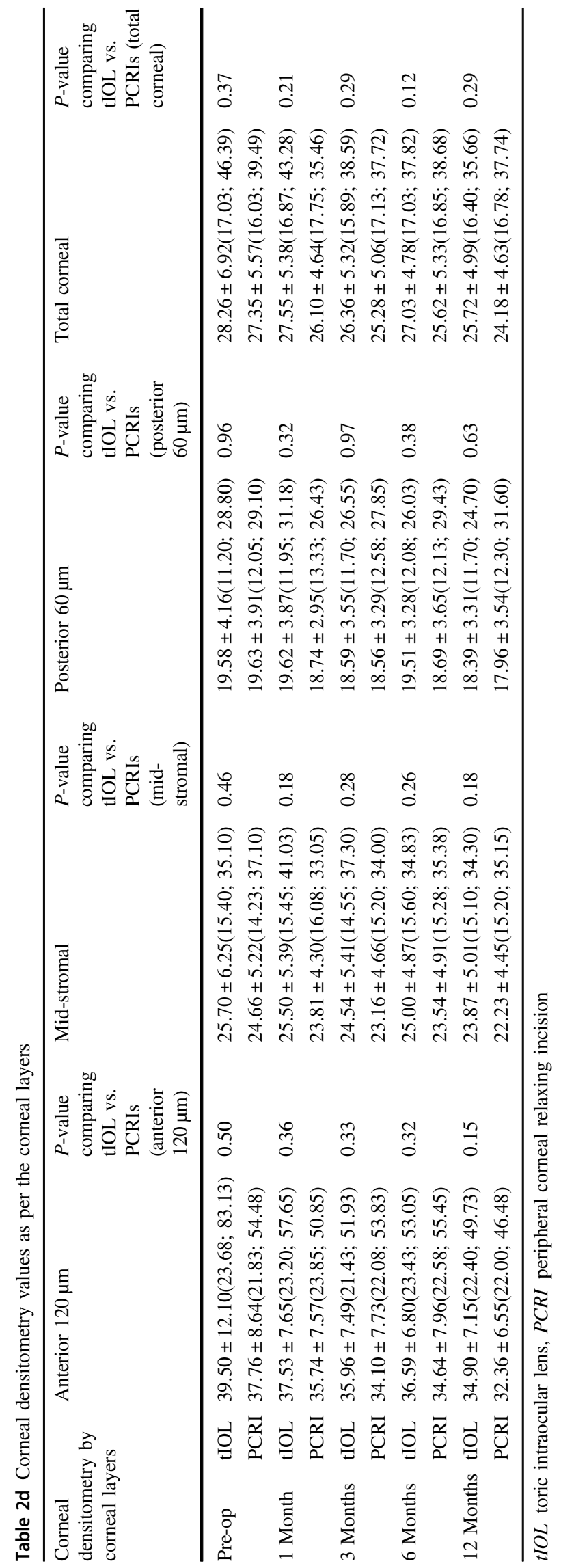


Table 3 Corneal densitometry comparison data taken from Poyales study [21] and our results at pre-operative and 3 months follow-up visits

\begin{tabular}{llllll}
\hline & LASIK-FS $^{\mathrm{a}}$ & PRK $^{\mathrm{a}}$ & ReLEx SMILE $^{\mathrm{a}}$ & tIOL & PCRI \\
\hline $\begin{array}{l}\text { Pre-operative } \\
\text { anterior120 } \mu \mathrm{m}\end{array}$ & $22.19 \pm 2.67$ & $22.20 \pm 2.49$ & $21.31 \pm 2.09$ & $39.50 \pm 12.10$ & $37.76 \pm 8.64$ \\
$\begin{array}{l}\text { Post-operative } \\
\text { anterior120 } \mu \mathrm{m}\end{array}$ & $22.14 \pm 2.82$ & $20.19 \pm 2.33$ & $20.98 \pm 2.10$ & $35.96 \pm 7.49$ & $34.10 \pm 7.73$ \\
$\begin{array}{l}\text { Pre-operative } \\
\text { mid-stromal }\end{array}$ & $14.74 \pm 1.77$ & $14.43 \pm 1.52$ & $14.55 \pm 1.32$ & $25.70 \pm 6.25$ & $24.66 \pm 5.22$ \\
$\begin{array}{l}\text { Post-operative } \\
\text { mid-stromal }\end{array}$ & $14.62 \pm 1.67$ & $14.07 \pm 1.53$ & $14.44 \pm 1.43$ & $24.54 \pm 5.41$ & $23.16 \pm 4.66$ \\
$\begin{array}{l}\text { Pre-operative } \\
\text { posterior60 } \mu \mathrm{m}\end{array}$ & $12.88 \pm \pm 1.72$ & $12.44 \pm 1.37$ & $13.00 \pm 1.23$ & $19.58 \pm 4.16$ & $19.63 \pm 3.91$ \\
$\begin{array}{l}\text { Post-operative } \\
\text { posterior60 } \mu \mathrm{m}\end{array}$ & $12.83 \pm 1.71$ & $12.34 \pm 1.46$ & $12.88 \pm 1.38$ & $18.59 \pm 3.55$ & $18.56 \pm 3.29$ \\
$\begin{array}{l}\text { Pre-operative total } \\
\text { Post-operative }\end{array}$ & $16.62 \pm 1.97$ & $16.38 \pm 1.67$ & $16.26 \pm 1.50$ & $28.26 \pm 6.92$ & $27.35 \pm 5.57$ \\
total & $16.53 \pm 1.94$ & $15.53 \pm 1.65$ & $16.10 \pm 1.54$ & $26.36 \pm 5.32$ & $25.28 \pm 5.06$ \\
\hline
\end{tabular}

LASIK-FS laser-assisted in situ keratomileusis with a femtosecond laser, $P R K$ photorefractive keratectomy, ReLEx SMILE ReLEx small-incision lenticule extraction, tIOL toric intraocular lens, PCRI peripheral corneal relaxing incision

${ }^{\mathrm{a} C}$ Corneal densitometry data taken from Poyales study [21] keratomileusis (LASIK) and small-incision lenticule extraction (SMILE) [21]. Two studies analysed corneal transparency after CXL over a longer time. Shen et al. [24] observed the corneal transparency over 1-year period founding unchanged densitometry values until 6-months follow-up, which decreased significantly after 12 months. Alnawaiseh et al. [23] found increase in corneal light backscatter 1 month after a standard epithelium-off CXL and decreased densitometry value to a baseline line at 12 months follow-up, which decreased significantly in the next 12-months follow-up. CXL reduces the corneal light backscatter significantly by increasing biomechanical strength of the cornea in patients with keratoconus [25].

Poyales et al. [21] was interested in corneal transparency changes occurring as a result of refractive surgery and chose to compare procedures as PRK, LASIK-FS and ReLex SMILE. The used measurement methods and statistical tests were similar to ours but their densitometry values were much lower due to the age of the participants. They found that the pre-operative spherical equivalent correlated to postoperative densitometry values whereas our results did not show any correlation. Poyales et al. [21] compared the preoperative $\mathrm{CD}$ values with 3-months follow-up results in PRK group finding significant results in all ring zones and layers when for ReLex SMILE, the significant decrease was found only in $10-12 \mathrm{~mm}$ zone and according to the anterior layer and total corneal thickness. For LASIK-FS intervention, significant changes were found only in $10-12 \mathrm{~mm}$ ring zone and no significant changes at any layer when compared by corneal depth. We compared our 3 months CD values for three layers of the cornea and total corneal CD with Poyales et al. [21] (Table 3). We observed our results to be higher than the CD values in the study by Poyales et al. [21]. We found that we had higher values of CD pre-operatively and this could be due to increased backscatter from cataracts compared to the younger population in their study. However, both ours and study by Poyales et al. [21], found higher $\mathrm{CD}$ values for anterior corneal layers. Boulze-Pankert et al. [26] has also analysed the impact of PRK on CD and found also a significant decrease at 3 months post-operatively. On the contrary, Lazaridis et al. [27] found increased CD values at 3 months after SMILE and decreased values of the posterior layer at statistically significant layer. In the FS-LASIK group, only the post-operative $\mathrm{CD}$ value of the posterior layer was found significantly decreased.

There are no other CD studies after cataract surgery with astigmatism correction up to $\leq 2.5 \mathrm{D}$ to compare our results with. However, it is interesting to note that, unlike excimer laser corneal refractive surgeries, PCRIs, which can correct astigmatism $\leq 2.5 \mathrm{D}$, does change the $\mathrm{CD}$ values as early as 1 month but there is no significant difference in the CD values over 1 year follow-up, compared to eyes without PCRIs (who had tIOLs). This study could be used as baseline for comparison to future studies.

In conclusion, the procedure of cataract surgery does reduce CD over 1 year follow-up in both tIOL and PCRI groups. PCRIs reduce the $\mathrm{CD}$ in peripheral corneal region more than tIOLs, although, not significant. PCRI shows significant changes in $\mathrm{CD}$ values 1 month onwards compared to 3 months onwards after no PCRI (tIOLs). 


\section{Study highlights}

\section{What was known before}

- Corneal densitometry could become an essential tool in post-operative monitoring protocols. At 3-months followup, corneal densitometry was not negatively affected by photorefractive keratectomy, laser in situ keratomeliousis and small-incision lenticule extraction.

\section{What this study adds}

- Cataract surgery significantly reduces the corneal densitometry due to reduction of backscatter During this 12-month follow-up study, cataract surgery combined with peripheral corneal relaxing incision (PCRIs) shows significant reduction in corneal densitometry from early post-operative period compared to later in eyes without PCRIs (when toric intraocular lenses were used). Changes in corneal densitometry are more significant in the central corneal zones and anterior corneal layer following cataract surgery.

\section{Compliance with ethical standards}

Conflict of interest The authors declare that they have no conflict of interest.

Publisher's note: Springer Nature remains neutral with regard to jurisdictional claims in published maps and institutional affiliations.

\section{References}

1. Maurice DM. The transparency of the corneal stroma. Vision Res. 1970;10:107-8.

2. Meek KM, Boote C. The organisation of collagen in the corneal stroma. Exp Eye Res. 2004;78:503-12.

3. Ni Dhubhghaill S, Rozema JJ, Jongenelen S, Ruiz Hidalgo I, Zakaria N, Tassignon MJ. Normative values for corneal densitometry analysis by Scheimpflug optical assessment. Invest Ophthalmol Vis Sci. 2014;55:162-8.

4. Otri AM, Fares U, Al-Aqaba MA, Dua HS. Corneal densitometry as an indicator of corneal health. Ophthalmology. 2012;119:501-8.

5. Moller-Pedersen T. A comparative study of human corneal keratocyte and endothelial cell density during aging. Cornea. 1997; 16:333-8.

6. Faragher RG, Mulholland B, Tuft SJ, Sandeman S, Khaw PT. Aging and the cornea. Br J Ophthalmol. 1997;81:814-7.

7. Hillenaar T, van Cleynenbreugel H, Remeijer L. How normal is the transparent cornea? Effects of aging on corneal morphology. Ophthalmology. 2012;119:241-8.

8. Bueno-Gimeno I, Espana-Gregori E, Gene-Sampedro A, Lanzagorta-Aresti A, Pinero-Llorens DP. Relationship among corneal biomechanics, refractive error, and axial length. Optom Vis Sci. 2014;91:507-13.

9. Shetty R, Francis M, Schroff R, Pahuja N, Khamar P, Girrish M, et al.Corneal biomechanical changes and tissue remodeling after SMILE and LASIK. Invest Ophthalmol Vis Sci. 2017;58:5703-12.

10. Freund DE, McCally RL, Farrell RA. Direct summation of fields for light scattering by fibrils with applications to normal corneas. Appl Opt. 1986;25:2739.

11. Smith GT, Brown NA, Shun-Shin GA. Light scatter from the central human cornea. Eye (Lond). 1990;4:584-8.

12. Nanavaty MA, Bedi KK, Ali S, Holmes M, Rajak S. Toric intraocular lenses versus peripheral corneal relaxing incisions for astigmatism between 0.75 and 2.5 diopters during cataract surgery. Am J Ophthalmol. 2017;108:165-77.

13. Tekin K, Inanc M, Kurnaz E, Bayramoglu E, Aydemir E, Koc M, et al. Objective evaluation of corneal and lens clarity in children with type 1 diabetes mellitus. Am J Ophthalmol. 2017;179:190-7.

14. Anayol MA, Bostancı B, Şekeroğlu MA, Şimşek M, Günaydın S, Yılmazbaş P. Assessment of corneal densitometry in rheumatoid arthritis patients. Turk J Ophthalmol. 2017;47:125-9.

15. Alnawaiseh M, Rosentreter A, Prokosch V, Eveslage M, Eter N, Zumhagen L. Changes in corneal densitometry in patients with fuchs endothelial dystrophy after endothelial keratoplasty. Curr Eye Res. 2017;42:163-7.

16. Lopes B, Ramos I, Ambrósio R. Corneal densitometry in keratoconus. Cornea. 2014;33:1282-6.

17. Alzahrani K, Carley F, Brahma A, Morley D, Hillarby MC. Corneal clarity measurements in healthy volunteers across different age groups: Observational study. Medicine (Baltim). 2017; 96:e8563.

18. Laule A, Cable MK, Hoffman CE, Hanna C. Endothelial cell population changes of human cornea during life. Arch Ophthalmol. 1978;96:2031-5.

19. Bourne WM, Nelson LR, Hodge DO. Central corneal endothelial cell changes over ten-year period. Invest Ophthalmol Vis Sci. 1997;38:779-82.

20. Garzón N, Poyales F, Illarramendi I, Mendicute J, Jáñez Ó, Caro $\mathrm{P}$, et al. Corneal densitometry and its correlation with age, pachymetry, corneal curvature, and refraction. Int Ophthalmol. 2017;37:1263-8.

21. Poyales F, Garzón N, Mendicute J, Illarramendi I, Caro P, Jáñez $\mathrm{O}$, et al. Corneal densitometry after photorefractive keratectomy, laser assisted in situ keratomileusis, and small-incision lenticule extraction. Eye (Lond). 2017;31:1647-54.

22. Jain R. Pentacam: principle and clinical applications. J Curr Glaucoma Pract. 2009;3:20-32.

23. Alnawaiseh M, Rosentreter A, Eveslage M, Eter N, Zumhagen L. Changes in corneal transparency after cross-linking for progressive keratoconus: Long-term follow-up. J Refract Surg. 2015; 31:614-8.

24. Shen Y, Jian W, Sun L, Li M, Han T, Son J, et al. One-year follow-up of changes in corneal densitometry after accelerated $(45 \mathrm{~mW} / \mathrm{cm} 2)$ transepithelial corneal collagen cross-linking for keratoconus: A retrospective study. Cornea. 2016;35:1434-40.

25. Spoerl E, Wollensak G, Seiler T. Increased resistance of crosslinked cornea against enzymatic digestion. Curr Eye Res. 2004; 29:35-40.

26. Boulze-Pankert M, Dariel R, Hoffart L. Corneal scheimpflug densitometry following photorefractive keratectomy in myopic eyes. J Refract Surg. 2016;32:788-91.

27. Lazaridis A, Droutsas K, Sekundo W, Petrak M, Schulze S. Corneal clarity and visual outcomes after small-incision lenticule extraction and comparison to femtosecond laser-assisted in situ keratomileusis. J Ophthalmol. 2017;2017:5646390. 